\title{
Examining the relationship between physical illness and depression: Is there a difference between inflammatory and non inflammatory diseases? A cohort study
}

\section{Running title: Higher risk of depression associated with Inflammatory disorders.}

\section{Authors}

1.- Constanza Caneo Robles, MD, MSc. corresponding author.

Psiquiatra acreditada. Departamento de Psiquiatría, Facultad de Medicina, Pontificia Universidad Católica de Chile. Chile.

Departamento de Psiquiatría Pontificia Universidad Católica de Chile. Diagonal Paraguay 476, segundo piso. Santiago Centro. Santiago.

CHILE. Postal code: 8330049 . Telephone: $+56-2-23543028 / /+56-9$ 42909250. Fax: 6651951

email: cmcaneo@uc.cl // constanza.caneo@gmail.com

2.- Michael King MD PhD.

Professor of Primary Care Psychiatry. Division of Psychiatry (Faculty of Brain Sciences), University College London. United Kingdom.

6th Floor, Maple House. 149 Tottenham Court Road. London, W1T 7NF, UK. Telephone: +44 (0)20 76799024

email: michael.king@ucl.ac.uk

3.- Louise Marston PhD.

Senior Research Associate. Department of Primary Care and Population Health, University College London. United Kingdom.

Rowland Hill Street, London. NW3 2PF, UK.

email: I.marston@ucl.ac.uk

4.- Juan Ángel Bellón

Profesor asociado. El Palo Health Centre, Primary Care District Málaga-Guadalhorce, Department of Preventive Medicine and Psychiatry,

University of Málaga and Biomedical Research Institute of Málaga

jabellon@uma.es

Word count:

- Article: 2917

- Abstract: 199.

Tables: 4 


\title{
Figures: 1
}

\section{Supplementary material: 4 tables}

\begin{abstract}
Background: There is evidence that inflammation may play a role in the association between physical illness and depression. Our aim was to compare the impact of chronic medical conditions on incidence of depression and to examine if risk of depression varies in terms of the presence and degree of inflammation.
\end{abstract}

Methods: This is a secondary analysis conducted within the Spanish sample of the predictD-study. Participants: 5,437. Primary outcome: Incident major depression measured with the Composite International Diagnostic Interview. Exposure: Presence of chronic medical conditions recorded by GPs using the International Classification of Primary Care, ICPC-2. All analyses were conducted using multivariable logistic regression to allow adjustment for confounders.

Results: The odds of depression are higher in almost all inflammatory than in non-inflammatory illnesses. There is an increasing risk of depression as a consequence of an increasing inflammatory load, with higher odds of depression in the autoimmune group than in the cardio-metabolic group, while both had higher odds of depression than the non-inflammatory groups.

Conclusions: Inflammation may be part of the pathway by which chronic physical illness leads to depression. Future studies should examine the role of inflammation in the prevention and management of depression.

Key words: Depression, inflammation, inflammatory disorders, chronic medical illnesses, major depressive disorder, cohort study. 


\section{INTRODUCTION}

There is a large body of evidence to support a bi-directional relationship between physical illness and depression. Observational studies have demonstrated that people with depression have higher rates of physical illness $(1,2)$, while patients with physical illness have higher rates of depression, a risk that follows a dose-response relationship with the number of concomitant physical illnesses (3-5).

In recent years substantial evidence has emerged regarding inflammation as one explanation for the physical illness-depression relationship (6-8). A longitudinal study with six-year follow-up showed, in an elderly depressionfree population, that systemic inflammatory states predicted the incidence of depression, an effect that persisted after adjustment for physical comorbidity, subjective wellbeing, use of antidepressants, and sociodemographic factors(9). Studies conducted with younger populations gave similar findings. The Avon Longitudinal Study of Parents and Children cohort (10) showed high levels of Interleukin 6 (IL-6) at age 9 predicted the incidence of depression at age 18, with a dose-response relationship, even after adjustment for sociodemographic factors and body mass index. Nonetheless, current evidence is based upon studies that adjusted for type of illness in the assessment of depression risk by inflammatory markers. Hence it was not possible to analyse and compare the clinical diagnoses in which inflammation occurred, which is relevant for the clinical implications of such findings. The systemic inflammatory response can be triggered by multiple stressors, both acute and chronic, and can be sustained for short or long periods of time, depending upon what triggered it. Hence a different approach for assessing the risk of depression in those with physical illness with an inflammatory profile is both scientifically and clinically necessary to improve current evidence about the illness-depression relationship.

The aim of this study was to explore and compare the impact of chronic medical conditions with and without an inflammatory profile on the incidence of depression. Our hypothesis was that chronic inflammatory illnesses confer 
a higher risk of depression than non-inflammatory illnesses, independently of the quality of life associated with the disease, in a population of primary care attendees free of depression at baseline.

\section{METHODS}

\subsection{Study design}

This is a secondary analysis with an a priori hypothesis, conducted within the completed Spanish cohort of the predictD study $(11,12)$, a cohort study with 12 months follow up to develop and validate a risk prediction algorithm for the onset of depression in primary care attendees. All participants in the Spanish sample accessed The Spanish National Health Service, which offers free access to medical care and covers $100 \%$ of the population, providing a representative sample of the population, with diverse economic backgrounds. The study population aged 18 to 75 years was recruited between October 2005 and February 2006. Participants were randomly selected from those booked to see their family physician each day. Between four and six attendees were selected daily. Exclusion criteria were inability to understand Spanish, or a diagnosis of psychosis, dementia or severe incapacitating physical illness. All risk factors for depression were measured at baseline (for more details see 11).

5,442 participants formed the predictD-Spain sample (12), but 95 participants had no information about their illness status, and were not included in our analysis, resulting in a final sample of 5,347 participants. At baseline, 741 patients had major depression (14\% of the sample), and thus were not included in the prediction of the risk of depression (See Figure 1).

The predictD-Spain study was approved by ethics committees in each Spanish province. Participants gave consent for using predictD data for subsequent research projects.

\subsection{Classification of inflammatory and non-inflammatory illnesses}

The main risk factor was the presence of chronic and inflammatory physical conditions. Conditions were recorded in the patients' medical records by their general practitioner (GP) using the International Classification of Primary Care, ICPC-2 of the World Association of Family Doctors (13). There were two main comparison groups: one defined as having no chronic medical condition, and the other as having only non-inflammatory disorders. Participants with absence of any chronic physical conditions were classified as "healthy". Thus, participants with 
acute, transient or non-specific physical conditions or complaints, such as influenza or sprained ankle were coded as healthy.

- 2.2.1 Chronic inflammatory conditions: Defined as physical illnesses that have a chronic course and feature, according to the literature, systemic inflammation as part of their pathophysiology $(7),(14),(15),(16),(17)$. This group includes cardiovascular illnesses, metabolic disorders and autoimmune disorders. The latter is the one that carries the most systemic inflammation.

- 2.2.2. Non inflammatory conditions: Osteoarthritis, respiratory disorders, allergies, peptic ulcer and gastroesophageal reflux disorder, functional disorders and locomotor pain complaints. Whilst asthma and chronic obstructive pulmonary disease (COPD), involve inflammation, they were coded as non-inflammatory as there is no clear evidence of systemic inflammation, but more of a locally situated response to pro-inflammatory substances such as tobacco smoke or air borne pollutants (18). The same principle was used for osteoarthritis, a clearly inflammatory but localised disorder, with no evidence of systemic inflammation.

- 2.2.3. Other unclassifiable disorders: Endocrine disorders were classified as a separate category because these disorders can involve inflammation but simultaneously hormonal alterations that independently increases the risk of depression. A similar criterion applied to neurological disorders, as there are other biological mechanisms than inflammation through which such disorders can induce or mimic depression (19).

For more detail about coding see appendix table 8.

\subsection{Measurement of major depression}

New cases of major depression were defined according to DSM-IV criteria using the depression section of the Composite International Diagnostic Interview (CIDI) (20) at either the six-month or 12-month follow-ups. Participants with missing outcome data at both follow-up times were excluded, as well as those with one missing outcome data who were not depressed in the assessment they attended, as we could not be certain that they had not experienced depression in the period with missing data. Participants with missing outcome data at one of the follow-up assessments and who had depression in the non-missed assessment attended were included in the analysis because they fulfilled the outcome criteria of depression during the one-year follow-up period. 


\subsection{Other possible explanatory variables (confounders)}

The confounders included in the final model were selected upon the evidence of an association with depression as described in predictD-study protocol (11), and also based on evidence of an association with chronic physical disorders, as described in the literature. To improve the precision in the estimation of the outcome, diagnoses of anxiety disorders and alcohol abuse were included in the adjusted model to control for comorbid psychiatric illnesses. Similarly, antidepressant or benzodiazepine use at baseline was included in the adjusted model to control for cases of treated depression.

- Sociodemographic characteristics: Age, gender, marital status, educational level, employment, financial strain, non-Spanish nationality.

- Psychosocial variables: Childhood experiences of physical, psychological or sexual abuse, sum of stressful life events in the past 6 month, experiences of discrimination for sex, age, ethnicity, appearance, disability or sexual orientation.

- Psychiatric antecedents and diagnosis at baseline: Self-rated mental and physical quality of life measured with SF-12(24), use of psychotropic medication according to medical records (antidepressants and benzodiazepines), alcohol misuse measured with Alcohol Use Disorders Identification Test (AUDIT)(25), and anxiety disorders measured using the anxiety item of the Primary Care Evaluation of Mental Disorders (PRIMEMD) (26). For more detail see appendix.

\subsection{Statistical analyses}

Baseline comparisons were made using descriptive statistics. The assessment of the risk of depression according to physical condition was conducted using multivariable logistic regression. The baseline characteristics of missing and successfully followed up participants were compared, together with an analysis of the association between these characteristics and missing status using the two sided independent t-test for continuous variables and Chi2 for binary variables to identify predictors of missing data. Sensitivity analyses were conducted to assess the impact of missingness on the estimation of the risk of depression. 
For multivariable analyses odds ratios and 95\% confidence intervals are reported. All analyses were conducted using Stata 12(21).

\section{RESULTS}

\subsection{Descriptive results}

Baseline characteristics of healthy participants and of the participants with the most prevalent diagnostic groups are displayed in Table 1. Quality of life (QoL) associated with physical health was low in those with autoimmune disorders, osteoarthritis, neurological and cerebrovascular disorders, but QoL associated with mental health was low in almost all groups (locomotor pain disorders, functional disorders, osteoarthritis, allergies, migraine, autoimmune disorder, endocrine, neurological, and cerebrovascular disorders).

There was a high comorbidity between diagnostic groups as shown in Table 2. It can be seen that no more than a third of the participants in each diagnostic group had just one diagnosis. The most frequent comorbid diagnoses were metabolic syndrome, cardiovascular disease, and osteoarthritis.

$3202(70 \%)$ of patients who were not depressed at baseline were successfully followed up at six months and 3015 $(66 \%)$ at 12 months (see Figure 1). After one year follow up, the cumulative incidence of depression in the total sample was $12 \%$ (321 new cases out of 2787 successfully followed up participants). The cumulative incidence of depression was $10 \%(124 / 1264)$ in healthy participants and $13 \%$ in participants with chronic physical conditions (197/1523). The incidence of depression in the autoimmune group was 20\% (12/61).

\subsection{Risk of depression}

The odds of depression were higher for all inflammatory illnesses before and after adjustment for confounders (Table 3). Most non-inflammatory illnesses showed no increased odds of depression, except for locomotor pain 
disorders (OR $2.2495 \% \mathrm{Cl} 1.36-3.71$ ), but this association was not statistically significant after adjustment for confounders (OR 1.57 95\% Cl $0.84-2.92)$.

We conducted a supplementary stepwise analysis (data not shown) to examine the increasing odds for depression in the cardiovascular group with adjustment. When we entered gender into the model no significant change occurred in the odds of depression (OR $1.3595 \% \mathrm{Cl} 1.01-1.81$ ), but when we added age a significant rise in the odds of depression occurred (OR $1.7995 \% \mathrm{Cl} 1.25-2.56$ ). Neither the point estimate nor the $95 \%$ confidence intervals changed after the addition of the remaining confounders.

Risk of depression rose with increasing inflammatory load, with higher odds of depression in the autoimmune than cardio-metabolic group, and both had higher odds of depression than the non-inflammatory group. After adjustment for illness load, the odds of depression still showed an increase with increasing inflammatory, but this finding was no longer statistically significant. The only group that had a significantly higher odds of depression after adjustment for illness load was the autoimmune disorder group (OR 2.43, 95\% $\mathrm{Cl} 1.05$ - 5.63) (Table 4, a).

In order to test whether comorbidity and illness load had a greater impact on risk of depression in the presence of inflammation, we stratified by comorbidity within the inflammatory illness group (Table 4, b). The regression model estimated that the odds of depression increased with inflammatory load. The highest odds of depression was associated with the presence of autoimmune disorders plus any other inflammatory disorder, which is our best proxy for a very high inflammatory state (OR 6.52, 95\% $\mathrm{Cl} 2.14-19.83)$. Similarly, the odds of depression in the groups with three or more cardio-metabolic disorders was higher than the odds where there was one cardiometabolic disorder (OR 2.38, 95\% Cl 1.22 - 4.61 for more than three cardio-metabolic disorders versus an OR of 1.28, $95 \% \mathrm{Cl} 0.84-1.97$ for one cardio-metabolic disorder).

\subsection{Missingness}

We examined the people with missing data on depression at one or both of the two follow up interviews. A baseline comparison of participants lost to follow up versus those successfully followed up, showed that the follow-up data were missing at random (see Appendix, Table 5). The pattern of missing data at baseline and each follow-up were as follows: no depression/missing/missing=1151 participants, no depression/missing/no depression=234 
participants, and no depression/no depression/missing=397 participants. Considering that data were missing at random and that covariates predicting missingness were included in the adjusted model, we undertook a complete case analysis. However, in a sensitivity analysis we assumed that all missing were either depressed or not depressed. In the first scenario, assuming missing participants as non-depressed (Appendix, Table 6), the cumulative incidence of depression decreased from $11.5 \%$ to $7.0 \%$. Nevertheless, the influence of illness category remained significant and followed the same increasing risk with illness category seen in the complete case analysis. In the second scenario when all missing were assigned a diagnosis of depression, the cumulative incidence rates rose to $22.6 \%, 27.9 \%$, and $46.0 \%$ respectively (Appendix, Table 7 ) and the associations with inflammation lost statistical significance.

\section{DISCUSSION}

The results of this secondary analysis of the predictD-Spain cohort study provides evidence for a higher risk of depression among people with physical illnesses characterized by systemic inflammation than in those with noninflammatory illnesses. Both load and type of illness impacted in a different manner upon the risk of depression, for example in the risk of depression conferred by comorbid cardio-metabolic versus autoimmune disorders. Hence, the assessment of physical illness as a risk factor for depression should take account of at least the type of comorbidity and number of illnesses involved.

The role of inflammation in major depression suggests new alternatives for treatment, especially in medically ill people. For instance, in a retrospective cohort study, Pasco et al found that both statins and aspirin are protective factors for depression (hazard ratios for depression $=0.20,95 \% \mathrm{Cl} 0.04-0.85)(22)$ in a population of long term users of these medications. A recently published meta-analysis of randomized controlled trials (23) concerning the role of anti-inflammatory medications in depression and depressive symptoms concluded that non-steroidal antiinflammatory drugs (NSAIDs) had a significant impact upon both depressive symptoms and remission of a major depressive episode. It is important to note that the exact nature of the physical health of the participants included in the studies was not clear, and there was only one study using healthy individuals. Thus we have little evidence of the effectiveness of NSAIDs or cytokine inhibitors in individuals with no physical illness, and it is not clear which specific clinical population might benefit from them. The role of anti-inflammatory drugs in depressive symptoms 
may be relevant only in populations suffering physical illnesses with an underlying inflammatory pathophysiology but, again, such a hypothesis has yet to be tested.

There are important limitations to consider in the interpretation of the findings. Firstly, we had to depend upon the variables that were available in the predictD-Spain data, thus excluding from the analysis risk factors that are relevant for the assessment of the illness-depression relationship, such as exercise, diet, and smoking behaviour. With regard to the latter, whilst it was taken into account in the predictD-study, the Spanish data had considerable missing data. Thus it was not possible to include it in our models. Secondly, the estimation of exposure to inflammation was assumed to be present according to diagnosis, without information about the level of inflammation, duration of exposure, severity of the illness, prognosis, and current treatment. One of the objectives of this study was to examine the role of inflammation in the context of diagnosis and comorbidity. However, in the absence of biological markers, interpretation of the role of inflammation remains theoretical, and we acknowledge this as a limitation to the interpretation of our results. Future studies should incorporate both biological markers and diagnostic criteria (such as ICD-10 or ICPC-2) to improve internal validity without compromising the generalizability of the findings. Moreover, the assessment of the impact of physical illness on the risk of depression should incorporate an comprehensive assessment of health, including disease diagnostic criteria, behavioural health factors and biological markers. A simple binary assessment of the presence or absence of any physical illness is likely to underestimate the impact that physical health has on psychiatric outcomes.

One of the strengths of this study is that the results are highly generalizable. The diagnoses were retrieved from the GPs' records using an internationally validated codification for physical illness, therefore increasing the comparability of these results with findings from other studies. With regard to internal validity, missingness was examined in detail and handled appropriately, thus providing robust evidence for the estimation of the outcome.

To our knowledge, this is the first prospective study to test the inflammatory hypothesis of depression, which uses diagnosis as a proxy for exposure to systemic inflammation, and considers the role of comorbidity in the impact of physical health on incidence of depression. Our results provide clinically relevant information about how the risk of depression varies according to type of illness and comorbidity, and suggest new research strategies for the assessment of illness as a risk factor for depression. 


\section{AKNOWLEDGEMENTS}

PredictD Spain was supported in Spain by grants from the Spanish Ministry of Health (grant FIS references: PI041980, PI041771, PI042450 and PI06/1442) ; the Andalusian Council of Health (grant references : 05/403 and 06/278) and the Spanish Ministry of Education and Science (grant reference SAF 2006/ 07192); the Spanish Network of Primary Care Research "redIAPP" (RD06/0018), the "Aragón group" (RD06/0018/0020), the "Baleares group" (RD07/0018/ 0033), and the "SAMSERAP group" (RD06/0018/ 0039). The Málaga sample, as part of the predictD- International study, was also co-funded by a grant from the European Commission (reference QL4CT2002-00683). The research in Europe was funded by a grant from the European Commission (reference PREDICT-QL4-CT2002-00683).

We would like to thank to MARISTÁN network (www.redmaristan.org), which was involved in the design, implementation and dissemination of the results of the predictD-study.

Constanza Caneo was awarded a University College London (UCL) Division of Psychiatry Scholarship, and BecasChile Scholarship to study a MSc. Mental Health Science Research at UCL, awarded by the National Commission for Scientific and Technological Research (CONICYT) of the Chilean Government.

\section{REFERENCES}

1. Smith DJ, Court H, McLean G, Martin D, Langan Martin J, Guthrie B, Gunn J, and Mercer SW. Depression and multimorbidity: A cross-sectional study of 1,751,841 patients in primary care. Journal of Clinical Psychiatry. 2014;75(11).

2. Aragonès E, Piñol JL, and Labad A. Depression and physical comorbidity in primary care. J Psychosom Res. England; 2007;63(2):107-11.

3. Kroenke K, Spitzer RL, Williams JB, Linzer M, Hahn SR, deGruy FV, and Brody D. Physical symptoms in primary care. Predictors of psychiatric disorders and functional impairment. Arch Fam Med. UNITED STATES; 1994;3(9):774-9. 
4. Patten SB. Long-term medical conditions and major depression in a Canadian population study at waves 1 and 2. Journal of Affective Disorders. 2001;63:35-41.

5. Stegmann ME, Ormel J, de Graaf R, Haro JM, de Girolamo G, Demyttenaere K, Kovess V, Matschinger H, Vilagut $\mathrm{G}$, Alonso J, Burger $\mathrm{H}$, and ESEMED/MHEDEA Investigators. Functional disability as an explanation of the associations between chronic physical conditions and 12-month major depressive episode. J Affect Disord. Netherlands; 2010;124(1-2):38-44.

6. Katon WJ. Clinical and health services relationships between major depression, depressive symptoms, and general medical illness. Biological Psychiatry. 2003;54(3):216-226.

7. Katon WJ. Epidemiology and treatment of depression in patients with chronic medical illness. Dialogues Clin Neurosci. France; 2011;13(1):7-23.

8. Purdy J. Chronic physical illness: a psychophysiological approach for chronic physical illness. Yale J Biol Med. United States; 2013;86(1):15-28.

9. Milaneschi Y, Corsi AM, Penninx BW, Bandinelli S, Guralnik JM, and Ferrucci L. Interleukin-1 receptor antagonist and incident depressive symptoms over 6 years in older persons: the InCHIANTI study. Biol Psychiatry. United States; 2009;65(11):973-8.

10. Khandaker GM, Pearson RM, Zammit S, Lewis G, and Jones PB. Association of serum interleukin 6 and Creactive protein in childhood with depression and psychosis in young adult life: a population-based longitudinal study. JAMA Psychiatry. United States; 2014;71(10):1121-8.

11. King M, Weich S, Torres-González F, Svab I, Maaroos HI, Neeleman J, Xavier M, Morris R, Walker C, BellónSaameño JA, Moreno-Küstner B, Rotar D, Rifel J, Aluoja A, Kalda R, Geerlings MI, Carraça I, de Almeida MC, Vicente B, Saldivia S, Rioseco P, and Nazareth I. Prediction of depression in European general practice attendees: the PREDICT study. BMC Public Health. England; 2006;6:6.

12. Bellón JÁ, de Dios Luna J, King M, Moreno-Küstner B, Nazareth I, Montón-Franco C, GildeGómez-Barragán MJ, Sánchez-Celaya M, Díaz-Barreiros MÁ, Vicens C, Cervilla JA, Svab I, Maaroos HI, Xavier M, Geerlings MI, Saldivia S, Gutiérrez B, Motrico E, Martínez-Cañavate MT, Oliván-Blázquez B, Sánchez-Artiaga MS, March S, del Mar Muñoz-García M, Vázquez-Medrano A, Moreno-Peral P, and Torres-González F. Predicting the onset of major depression in primary care: international validation of a risk prediction algorithm from Spain. Psychol Med. England; 2011;41(10):2075-88. 
13. Okkes IM, Jamoulle M, Lamberts H, and Bentzen N. ICPC-2-E: the electronic version of ICPC-2. Differences from the printed version and the consequences. Family Practice [[Internet]]. 2000;17(2):101-107. from: http://fampra.oxfordjournals.org/content/17/2/101.abstract

14. Krishnadas R, and Cavanagh J. Depression: an inflammatory illness? J Neurol Neurosurg Psychiatry. England; 2012;83(5):495-502.

15. Almond MA. Depression and Inflammation: Examining the link. Current Psychiatry. 2013;12(6):25-32.

16. Postal M, and Appenzeller S. The importance of cytokines and autoantibodies in depression. Autoimmun Rev. Netherlands; 2015;14(1):30-5.

17. Berk M, Williams LJ, Jacka FN, O'Neil A, Pasco JA, Moylan S, Allen NB, Stuart AL, Hayley AC, Byrne ML, and Maes M. So depression is an inflammatory disease, but where does the inflammation come from? BMC Med. England; 2013;11:200.

18. Bonsignore MR, Profita M, Gagliardo R, Riccobono L, Chiappara G, Pace E, and Gjomarkaj M. Advances in asthma pathophysiology: stepping forward from the Maurizio Vignola experience. Eur Respir Rev. England; 2015;24(135):30-9.

19. Chwastiak LA, and Ehde DM. Psychiatric issues in multiple sclerosis. Psychiatr Clin North Am. United States; 2007;30(4):803-17.

20. Robins LN, Wing J, Wittchen HU, Helzer JE, Babor TF, Burke J, Farmer A, Jablenski A, Pickens R, and Regier DA. The Composite International Diagnostic Interview. An epidemiologic Instrument suitable for use in conjunction with different diagnostic systems and in different cultures. Arch Gen Psychiatry. UNITED STATES; 1988;45(12):1069-77.

21. College Station, TX: StataCorp LP.. Stata Statistical Software: Release 12. StataCorp. 2011.

22. Pasco JA, Jacka FN, Williams LJ, Henry MJ, Nicholson GC, Kotowicz MA, and Berk M. Clinical implications of the cytokine hypothesis of depression: the association between use of statins and aspirin and the risk of major depression. Psychother Psychosom. Switzerland; 2010. p. 323-5.

23. Köhler O, Benros ME, Nordentoft M, Farkouh ME, lyengar RL, Mors O, and Krogh J. Effect of anti-inflammatory treatment on depression, depressive symptoms, and adverse effects: a systematic review and meta-analysis of randomized clinical trials. JAMA Psychiatry. United States; 2014;71(12):1381-91.

24. Ware J, Kosinski M, and Keller SD. A 12-Item Short-Form Health Survey: construction of scales and preliminary tests of reliability and validity. Med Care. UNITED STATES; 1996;34(3):220-33. 
25. Saunders JB, Aasland OG, Babor TF, de la Fuente JR, and Grant M. Development of the Alcohol Use Disorders Identification Test (AUDIT): WHO Collaborative Project on Early Detection of Persons with Harmful Alcohol Consumption--II. Addiction. ENGLAND; 1993;88(6):791-804.

26. Spitzer RL, Williams JB, Kroenke K, Linzer M, deGruy FV, Hahn SR, Brody D, and Johnson JG. Utility of a new procedure for diagnosing mental disorders in primary care. The PRIME-MD 1000 study. JAMA. UNITED STATES; 1994;272(22):1749-56. 